\title{
Morfologia duplex Doppler dos principais vasos sanguíneos abdominais em pequenos animais
}

\author{
Duplex Doppler morphology of major abdominal blood vessels in small animals
}

\author{
Cibele Figueira Carvalho ${ }^{\mathrm{I}, \mathrm{II}}$ Maria Cristina Chammas ${ }^{\mathrm{II}}$ Giovanni Guido Cerri ${ }^{\mathrm{II}}$
}

\section{- REVISÃo BIBLIOGRÁFICA -}

\section{RESUMO}

A ultra-sonografia duplex Doppler é capaz de fornecer informações anatômicas e hemodinâmicas em tempo real. O conhecimento do espectro Doppler normal de cada vaso sanguíneo é importante na sua identificação, pois cada vaso possui um sinal Doppler específico. Reconhecer as alterações de morfologia do espectro somente é possível mediante o conhecimento das variações na normalidade. $O$ objetivo deste artigo foi revisar as informações publicadas em literatura que descrevem o padrão Doppler normal dos principais vasos sanguíneos abdominais de pequenos animais.

Palavras-chave: ultra-sonografia, duplex Doppler, vascularização, abdômen.

\section{ABSTRACT}

Duplex Doppler ultrasonography is able to provide real time anatomic and hemodynamic information. The complete knowledge of the normal Doppler spectrum of each blood vessel is important to their identification, since each vessel has a specific Doppler sign. Recognizing the changes in the morphology of Doppler spectrum is only possible based on previous knowledge of its variations. This is a literature review about Doppler pattern of the major abdominal blood vessels in small animals.

Key words: ultrasonography, duplex Doppler, vascularization, abdomen.

\section{INTRODUÇÃO}

A ultra-sonografia Doppler é uma ferramenta diagnóstica recente na Medicina Veterinária de pequenos animais. A ultra-sonografia duplex Doppler é capaz de fornecer informações em tempo real sobre a arquitetura vascular e os parâmetros hemodinâmicos dos vasos. O conhecimento dos traçados Doppler normais de cada vaso sanguíneo é importante na detecção e identificação de cada um deles, no reconhecimento das alterações resultantes de patologias evidenciadas pela presença ou ausência de fluxo sanguíneo no vaso supostamente acometido. O objetivo deste artigo foi revisar as informações publicadas em literatura que descrevem o padrão Doppler normal dos principais vasos sanguíneos abdominais de pequenos animais, diante da escassez de artigos publicados sobre o tema.

\section{Anatomia geral ecográfica}

A ultra-sonografia bidimensional convencional fornece informações sobre a localização de vasos, a avaliação do diâmetro, a espessura e regularidade das paredes vasculares ou a presença de estruturas anormais perivasculares ou intraluminais. Em geral, as estruturas anormais têm ecogenicidade diferente do conteúdo do vaso. Porém, alguns trombos recentes podem ser anecóides e sua detecção depende da avaliação Doppler do fluxo sangüíneo no vaso avaliado (FINN-BODNER \& HUDSON, 1998).

Os vasos sangüíneos abdominais têm estrutura tubular com paredes finas e bem definidas em plano longitudinal. As paredes são paralelas

\footnotetext{
IServiço de ultra-sonografia, Instituto Brasileiro de Diagnóstico e Especialidades Veterinárias (PROVET). Travessa Leon Berry, 122, Jardim Paulista, 01402-030, São Paulo, SP, Brasil. E-mail: cibelefcarvalho@terra.com.br. Autor para correspondência.

"Instituto de Radiologia do Hospital das Clínicas, Faculdade de Medicina, Universidade de São Paulo (USP), São Paulo, SP, Brasil.
} 
hiperecóides e com aparência linear. Em plano transversal, os vasos podem aparecer com aspecto oval ou circular e alguns sofrem alteração na morfologia quando submetidos à compressão (SPAULDING, 1997). A presença de sangue no lúmen confere ao conteúdo vascular um aspecto anecóide, característica das estruturas que não possuem eco, isto é, que não transmitem onda sonora. Porém, quando o fluxo sangüíneo é lento e o diâmetro do vaso é grande o suficiente, podem-se observar pontos hiperecóides que se movimentam e correspondem às células sanguíneas (NYLAND \& MATTOON, 2002).

Durante o exame de um vaso sangüíneo, inicialmente observa-se o vaso usando-se a técnica bidimensional e obtendo-se um plano longitudinal do mesmo. O eixo do feixe ultra-sônico e o eixo do vaso devem estar paralelos entre si, porém o ângulo de insonação (ângulo formado entre a parede vascular e o cursor Doppler) não deve ser maior que $60^{\circ}$ (CERRI et al., 1998; YANIK, 2002). Isto é importante, pois os parâmetros velocimétricos do sangue calculados pela técnica Doppler são ângulodependente (Figura 1).

Usando-se o modo Doppler colorido, é possível determinar-se a presença ou a ausência de fluxo no vaso. Os parâmetros coloridos devem ser ajustados para que o lúmen do vaso esteja preenchido somente com uma cor (isto é, sem ambigüidade de sinal

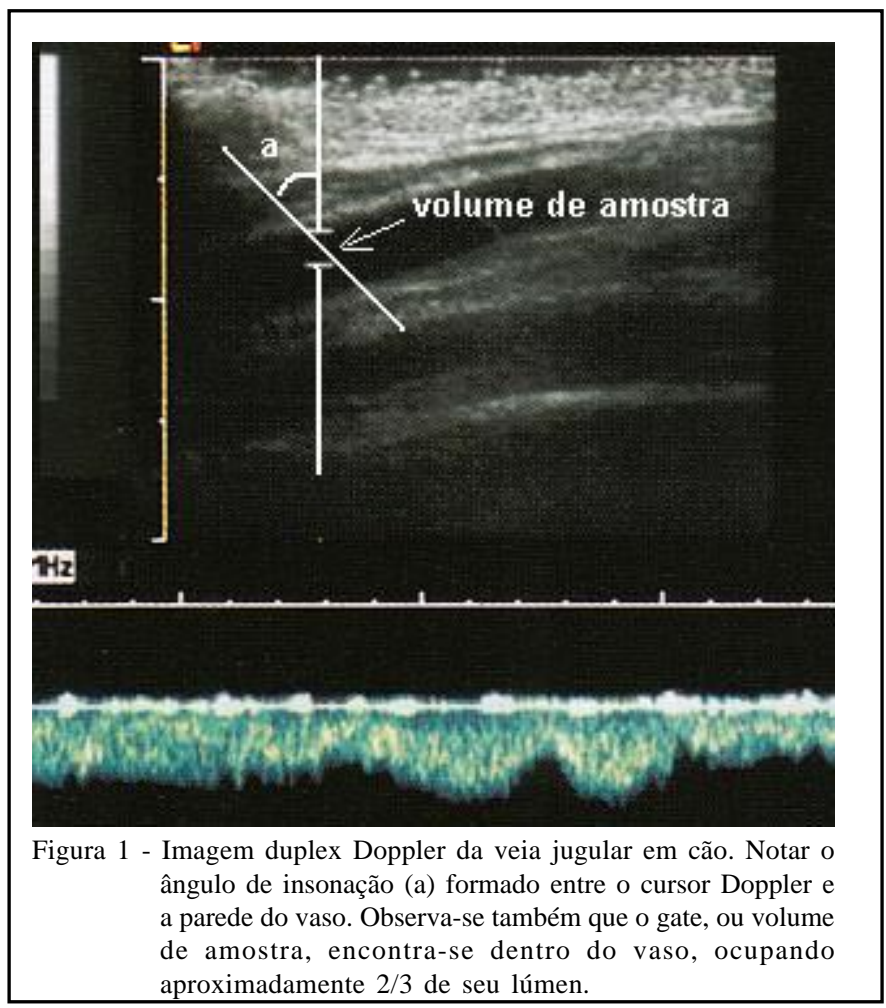

detectado) e a informação colorida não ultrapasse o lúmen vascular, mas que o mesmo esteja todo preenchido com cor (CERRI et al., 1998; KAWAKAMA et al., 1993). Por fim, o volume de amostra ou gate deve ser colocado em uma porção específica do vaso, em geral na região central, ocupando até $2 / 3$ do diâmetro (Figura 1). Usando-se a técnica Doppler pulsado, um traçado pode ser obtido nesta região particular do vaso. Se o traçado Doppler estiver livre de artefatos, a imagem deverá ser congelada e, após correção do ângulo, deve-se proceder à análise da morfologia das ondas (CERRI et al., 1998).

Cada vaso possui uma assinatura particular, ou seja, um traçado característico, que permite sua identificação e a observação de alterações patológicas.

\section{Aorta abdominal}

A aorta passa a ser chamada abdominal à medida que ingressa na porção dorsocranial desta cavidade, atravessando o hiato aórtico, dorsal ao diafragma e no plano médio sagital da cavidade retroperitoneal (CARVALHO, 2004). Amelhor imagem da aorta abdominal em sua porção caudal é obtida com o animal em decúbito lateral direito. O transdutor é colocado na região caudodorsal do abdome, onde a pulsação da aorta pode ser observada longitudinalmente, em posição ventral às vértebras lombares, à esquerda e paralela ao trajeto da veia cava caudal. A parte cranial da aorta abdominal (cranial à artéria frenicoabdominal esquerda) não é de fácil visibilização, especialmente em cães com tórax profundo, devido aos artefatos ocasionados pelo gás no trato gastrintestinal e pelas costelas. Às vezes, quando há muito gás, pode ser mais fácil obterse uma imagem sagital da região cranial da aorta em decúbito dorsal, medialmente ao rim direito (KAMIKAWA, 2003; SZATMARI et al., 2001). A aorta possui um perfil típico de velocidade de fluxo laminar do tipo achatado e a morfologia da onda é típica de um fluxo de padrão de alta resistividade. Possui pico sistólico afilado, com uma janela espectral larga e bem definida (Figura 2). A distribuição de velocidade é estreita. Pode ainda haver variação na velocidade de fluxo sangüíneo, dependendo da região abdominal na qual foi efetuada a avaliação. O pico sistólico é seguido por uma onda de fluxo retrógrado; depois, pode ser vista uma onda de fluxo anterógrada (CERRI et al.,1998). Se houver uma pausa maior entre duas contrações ventriculares, pode-se ainda observar ondas adicionais com velocidades mais baixas (SZATMARI et al., 2001). 


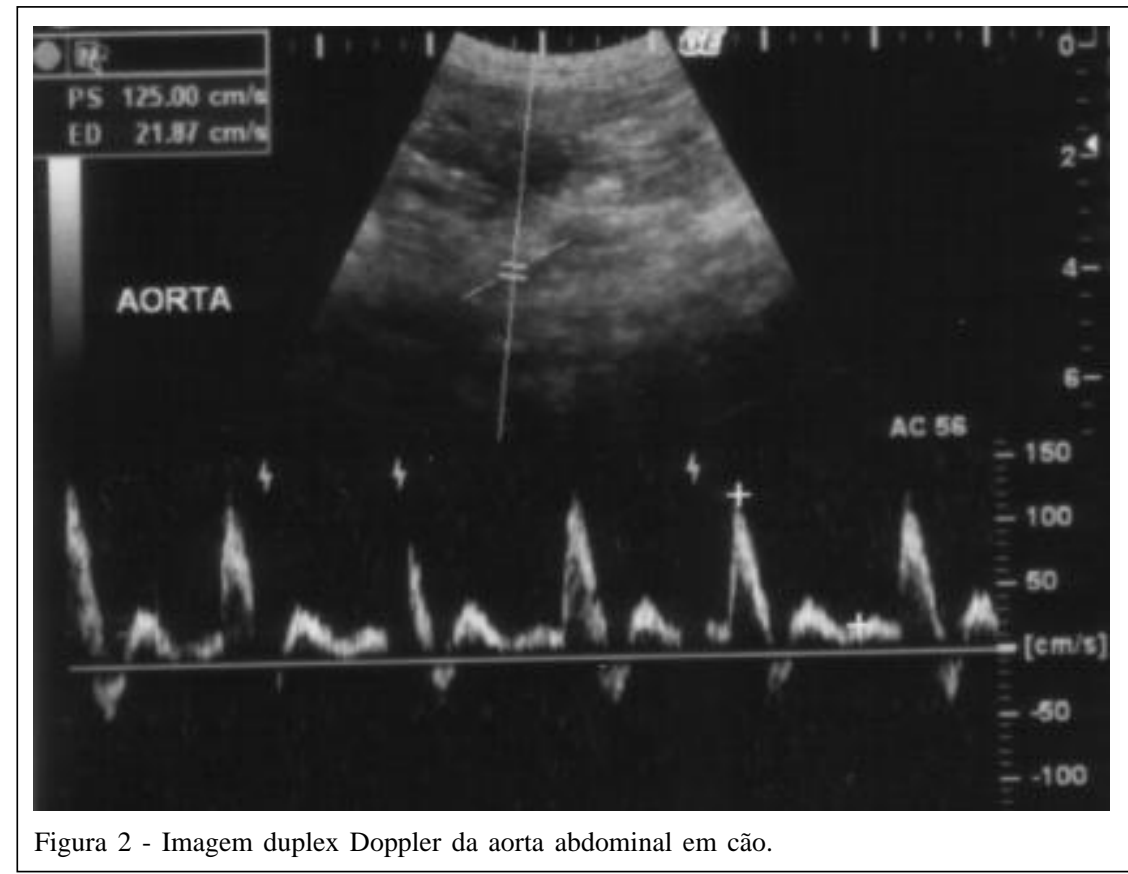

Artérias ilíacas internas

As artérias ilíacas internas direita e esquerda são os últimos ramos da aorta. Elas são responsáveis pela irrigação da pelve e se originam simetricamente da superfície lateral da aorta. Caudal a este ponto, a aorta torna-se a artéria sacral mediana (KAMIKAWA, 2003). Sugere-se o decúbito lateral para avaliá-las, pois nesta posição há menor atenuação do feixe sonoro pelos tecidos superficiais. Pode-se detectar um perfil de velocidade de fluxo semiparabólico e com padrão de alta resistividade. Observam-se picos sistólicos afilados, com uma janela espectral muito pequena. Há uma pequena quantidade de fluxo retrógrado protodiastólico; depois, o fluxo torna-se anterógrado no resto da diástole (SZATMARI et al., 2001). Esta característica é facilmente visibilizada através de uma pequena depressão no traçado espectral antes do início da diástole.

\section{Artéria mesentérica cranial}

Este vaso ímpar é o segundo ramo da aorta abdominal. Sua origem é ligeiramente caudal à origem da artéria renal direita e um pouco caudal à do tronco celíaco. Se o animal estiver em decúbito dorsal, o tronco celíaco e a artéria mesentérica cranial formam um "V" no plano de corte sagital (Figura 3). Quando o cão estiver em decúbito lateral direito, o tronco celíaco e a artéria mesentérica cranial são vasos de trajeto paralelo, que se tocam (GASCHEN et al., 2005). Pode ser observado perfil de velocidade de fluxo laminar do tipo semiparabólico e um padrão de fluxo de resistividade intermediária. O pico sistólico é mais amplo com uma janela espectral pequena. Não há fluxo diastólico reverso. Após o pico sistólico, a velocidade cai bruscamente e, logo, o fluxo retorna mais rápido; depois, mais lento novamente (Figura 3). O sangue apresenta maior resistência no período pós-prandial (RIESEN et al., 2002).

\section{Artérias e veias renais}

A artéria e a veia renal podem ser observadas desde a região hilar renal até sua origem na aorta e na veia cava caudal, respectivamente. Os ramos interlobares podem ser vistos ao redor do complexo ecogênico central, irradiando-se da pelve em direção à junção córtico-medular. As artérias interlobulares originam-se das artérias arqueadas e tornam-se visíveis usando-se Doppler colorido. As veias geralmente são mais largas do que as artérias adjacentes, e ambas correm paralelas (NYLAND et al., 1993).

As artérias renais têm perfil típico de velocidade de fluxo parabólico (isto é, picos sistólicos com ampla distribuição de velocidade e sem janela espectral). O pico sistólico é sempre afilado e, algumas vezes, observa-se a presença de incisura protodiastólica (ou seja, pode aparecer uma pequena depressão no traçado espectral antes do início da diástole). Baixa resistividade de fluxo pode ser detectada com fluxo diastólico contínuo e cheio, que gradualmente diminui durante a diástole (Figura 4). Após o pico sistólico, a velocidade cai um pouco; depois, torna-se mais alta novamente (pico de 

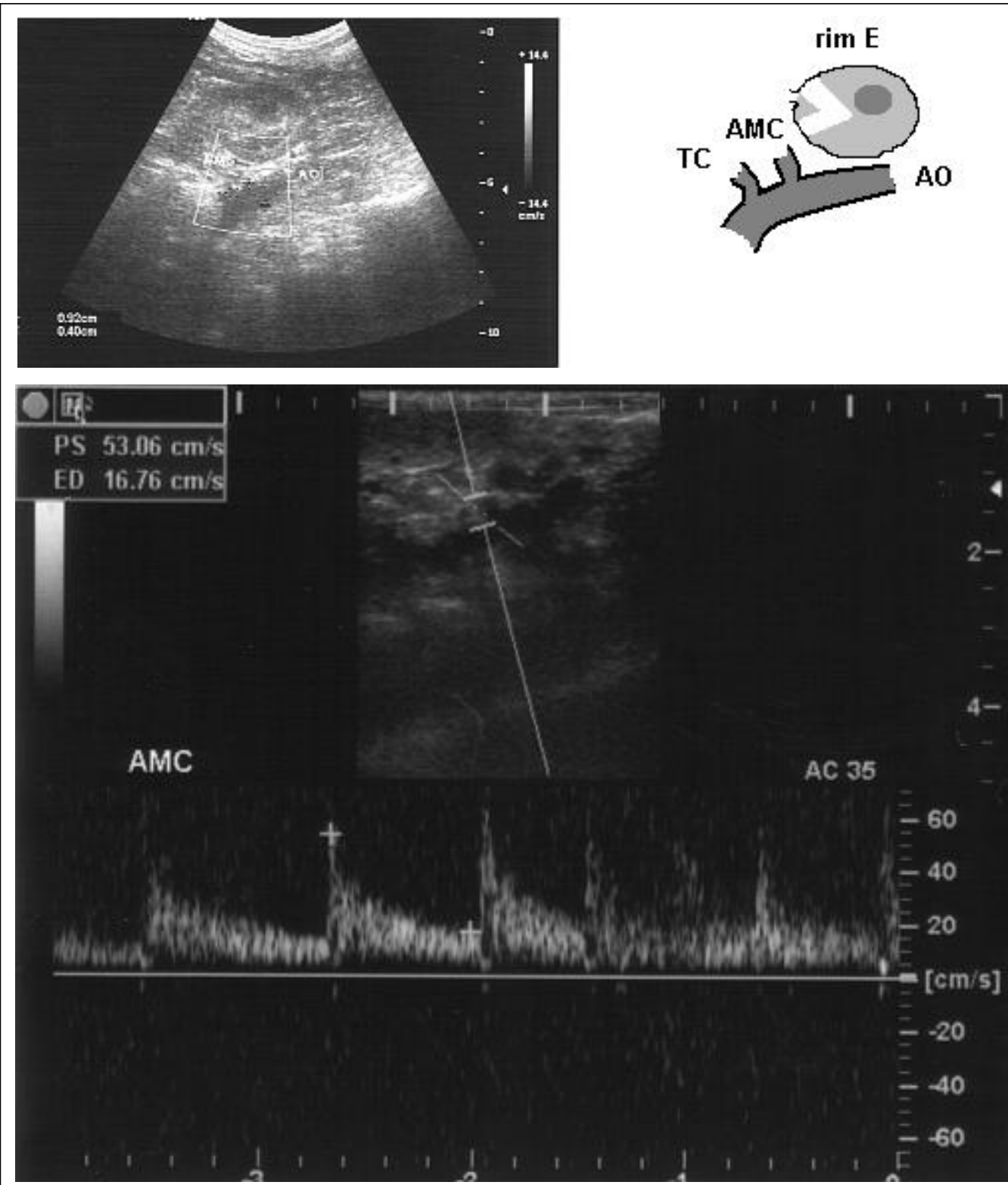

Figura 3 - A) Imagem duplex Doppler colorido com desenho esquemático mostrando plano de corte sagital de tronco celíaco (TC) e a artéria mesentérica cranial (AMC), ambas se originando da aorta abdominal (AO) em região cranial ao rim esquerdo (rim E). B) Imagem duplex Doppler da artéria mesentérica cranial (AMC) em cão.

velocidade diastólico) e, no restante da diástole, gradualmente diminui (CERRI et al., 1998; SZATMARI et al., 2001).

\section{Tronco celíaco}

Este é o primeiro ramo ímpar da aorta abdominal. Após um tronco curto, distribui-se em três artérias (tripus Halleri): a artéria hepática comum, a esplênica e a gástrica esquerda. A melhor imagem pode ser obtida em decúbito dorsal ou lateral direito, medial ao rim direito, embora o gás do duodeno freqüentemente dificulte a imagem. A imagem é mais difícil de se obter em cães de tórax profundo e afilado (GASCHEN et al., 2005).

Pode-se detectar perfil de velocidade de fluxo semiparabólico e padrão de fluxo de resistividade intermediária. Podem-se ver picos sistólicos amplos com janela espectral de tamanho médio e evidente. Não se observa fluxo diastólico reverso. A velocidade durante a diástole é alternante, semelhante ao padrão 


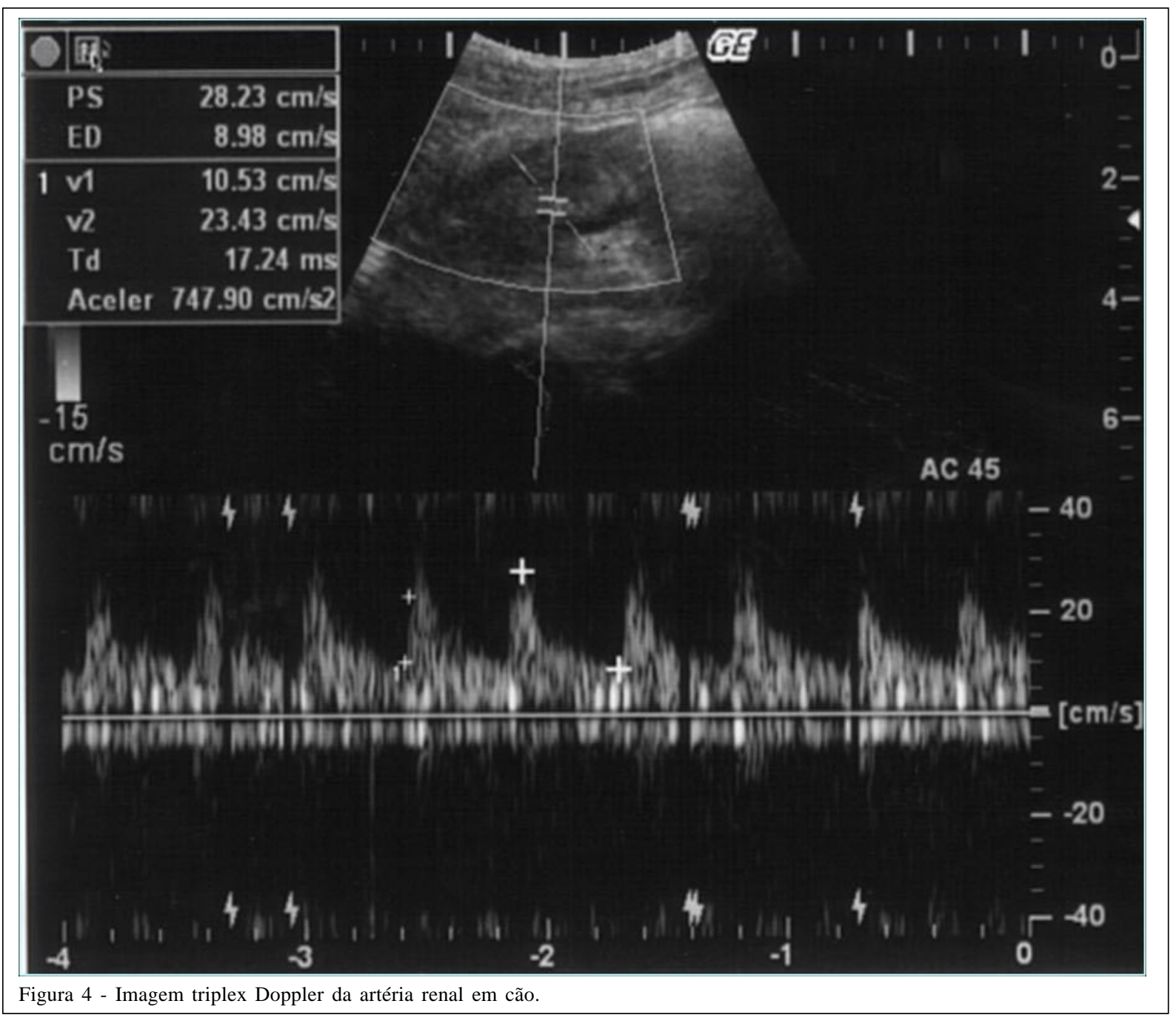

de outras artérias com padrão de fluxo de resistividade intermediária. Em alguns cães, pode-se ver a presença de picos sistólicos dicróicos (SZATMARI et al., 2001), ou seja, traçado espectral com picos sistólicos duplos.

\section{Artéria hepática}

A artéria hepática comum, na maioria das vezes, pode ser vista somente com a utilização do Doppler colorido, uma vez que se origina cranialmente do tronco celíaco, próximo da veia porta principal. Corre paralela entre a veia cava caudal e a veia porta. Os ramos intra-hepáticos da artéria hepática geralmente não podem ser encontrados em cães sadios, devido ao seu pequeno tamanho (LAMB et al., 1999). Seu padrão de espectro Doppler (Figura 5) é semelhante ao espectro da artéria renal (isto é, perfil de fluxo parabólico e padrão de fluxo de baixa resistividade). Podem ser detectados picos sistólicos amplos e contínuos sem janela espectral (ampla distribuição de velocidades), com fluxo diastólico alto, que diminui gradualmente (CERRI et al., 1998; SZATMARI et al., 2001).
Artéria e veia esplênica

As veias esplênicas podem ser vistas ao exame ecográfico modo-B de rotina, identificadas como uma interrupção na continuidade da cápsula do baço, mas as artérias esplênicas, muito finas, podem ser vistas somente com Doppler colorido, adjacentes às veias. A artéria esplênica principal se origina do tronco celíaco e termina ramificando-se no hilo esplênico, mas, devido a seu curso tortuoso e do gás no trato intestinal, sua imagem raramente é contínua desde o tronco celíaco até o baço. A veia esplênica principal e a artéria podem ser vistas no hilo esplênico. A veia esplênica flui para a veia porta. Podemos encontrar uma morfologia de onda típica de fluxo de baixa resistividade (isto é, picos sistólicos amplos e contínuos com fluxo diastólico alto ou diástole cheia), assim como perfil de velocidade de fluxo parabólico (isto é, picos sistólicos sem janela espectral) semelhante ao padrão espectral Doppler das artérias renais e hepática (Figura 6). Em alguns animais, 


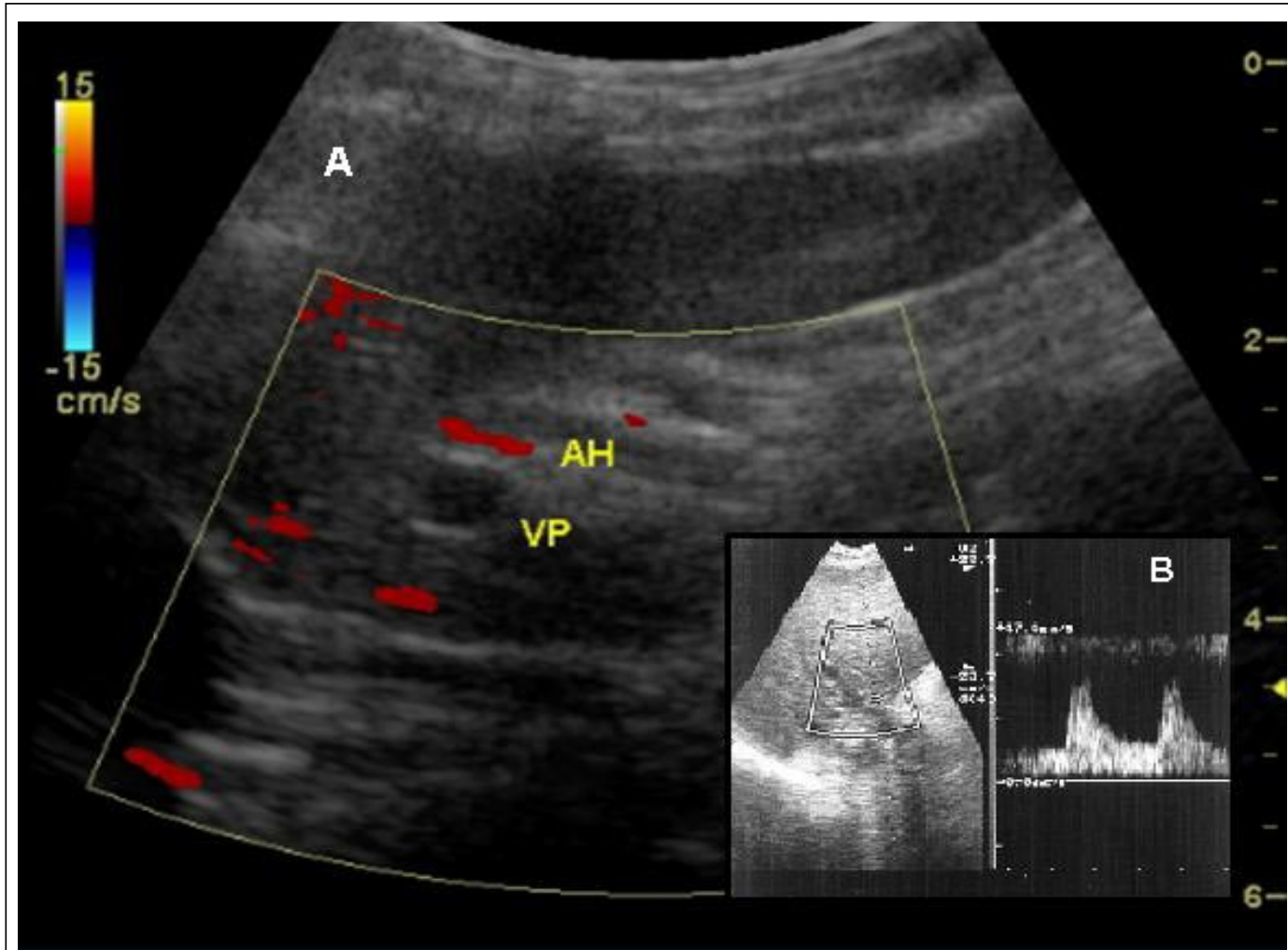

Figura 5 - A) Imagem duplex Doppler colorido de artéria hepática (AH) em cão. VP = veia porta. B) imagem duplex Doppler de artéria hepática mostrando o traçado espectral da mesma.

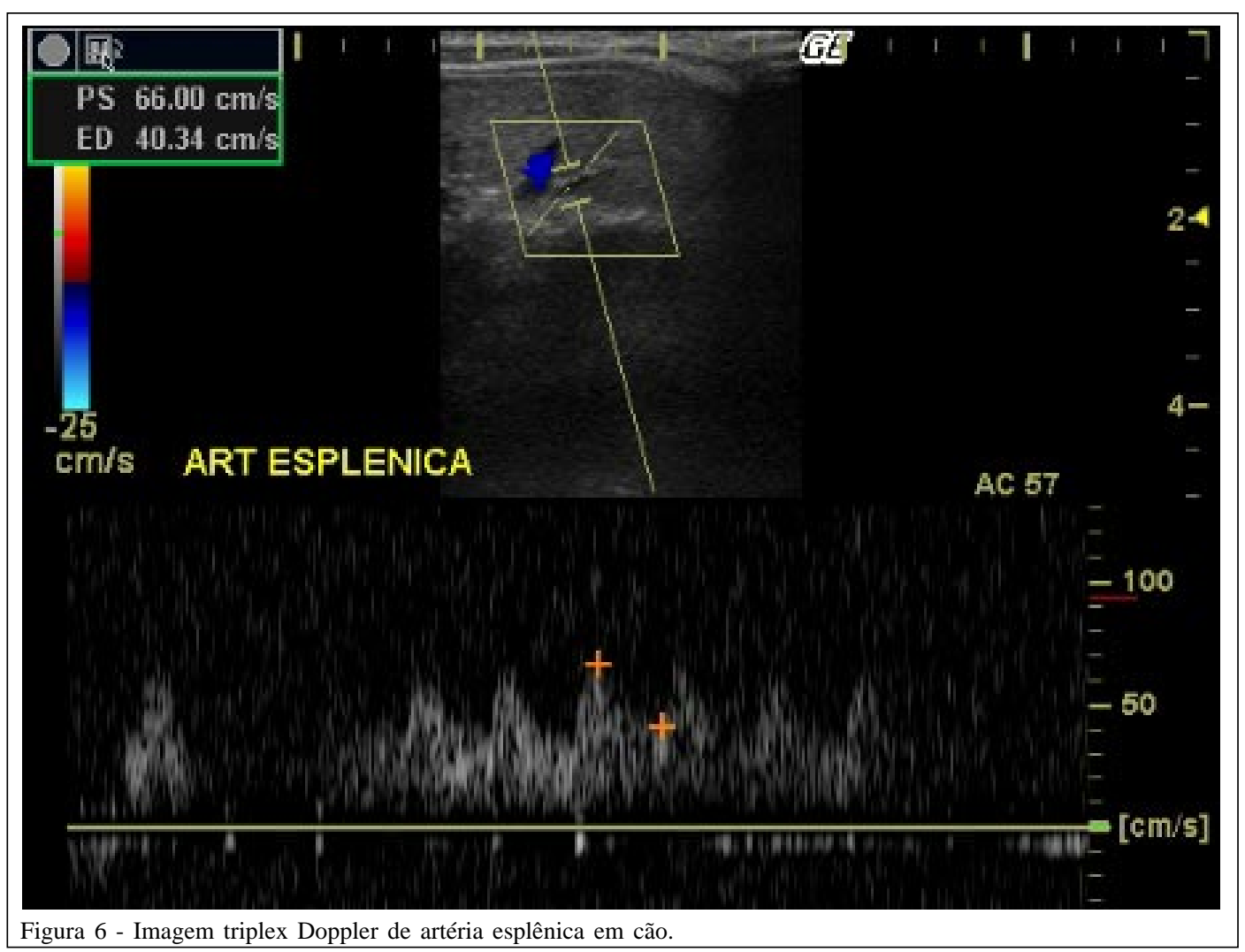

Ciência Rural, v.38, n.3, mai-jun, 2008. 
pode estar presente incisura protodiastólica (SZATMARI et al., 2001).

\section{Veia cava caudal}

A parte caudal da veia cava encontra-se adjacente à aorta abdominal. Elas têm aproximadamente o mesmo diâmetro. Cranial à entrada das veias renais, a veia cava caudal desvia-se na direção cranioventral e entra na cavidade torácica através do diafragma. A parte cranial da veia cava caudal é mais fácil de ser escaneada em decúbito lateral esquerdo ou dorsal, medialmente ao rim direito. A veia cava caudal está sempre ao lado da veia porta e apresenta-se como um vaso retilíneo e paralelo neste ponto e que, depois, apresenta um curso curvilíneo. A veia porta principal é ventral e situa-se ligeiramente à esquerda da veia cava caudal (KAMIKAWA, 2003). Nesta parte cranial, a morfologia de onda é mais periódica e fásica do que em sua região caudal, correspondendo às condições de pressão do átrio direito, uma vez que as alterações de pressão intratorácica e intra-abdominal ocorrem devido aos movimentos respiratórios (CERRI et al., 1998). Observa-se que cada contração ventricular é seguida por uma onda de fluxo anterógrado que aumenta ligeiramente a velocidade (Figura 7). Além disso, cada movimento inspiratório causa uma onda de fluxo anterógrada de velocidade alta (CERRI et al., 1998; SZATMARI et al., 2001).

Veias hepáticas

As veias hepáticas e suas convergências na veia cava caudal podem ser vistas no fígado. A melhor imagem para o estudo Dopplervelocimétrico pode ser obtida em decúbito lateral esquerdo, em janela intercostal, na altura entre o décimo e o décimo primeiro espaço intercostal, no plano sagital. O número e a anatomia das veias hepáticas encontram-se descritos em literatura (WU \& CARLISLE,1995). O padrão espectral Doppler das veias hepáticas é fortemente periódico, e as ondas correspondem à pressão atrial direita (Figura 8). A terminologia utilizada para descrever a morfologia das ondas é baseada em referências médicas com estudos no homem (CERRI et al., 1998).

A primeira onda é anterógrada e larga (ondaS) e é causada pelo movimento de fechamento da tricúspide em direção ao ápice cardíaco. O segundo componente do espectro Doppler das veias hepáticas é uma onda pequena e retrógrada (onda-V) causada pelo preenchimento (diástole) atrial direito. O terceiro componente é a segunda onda anterógrada (onda-D), que é causada pela abertura da valva tricúspide e pelo

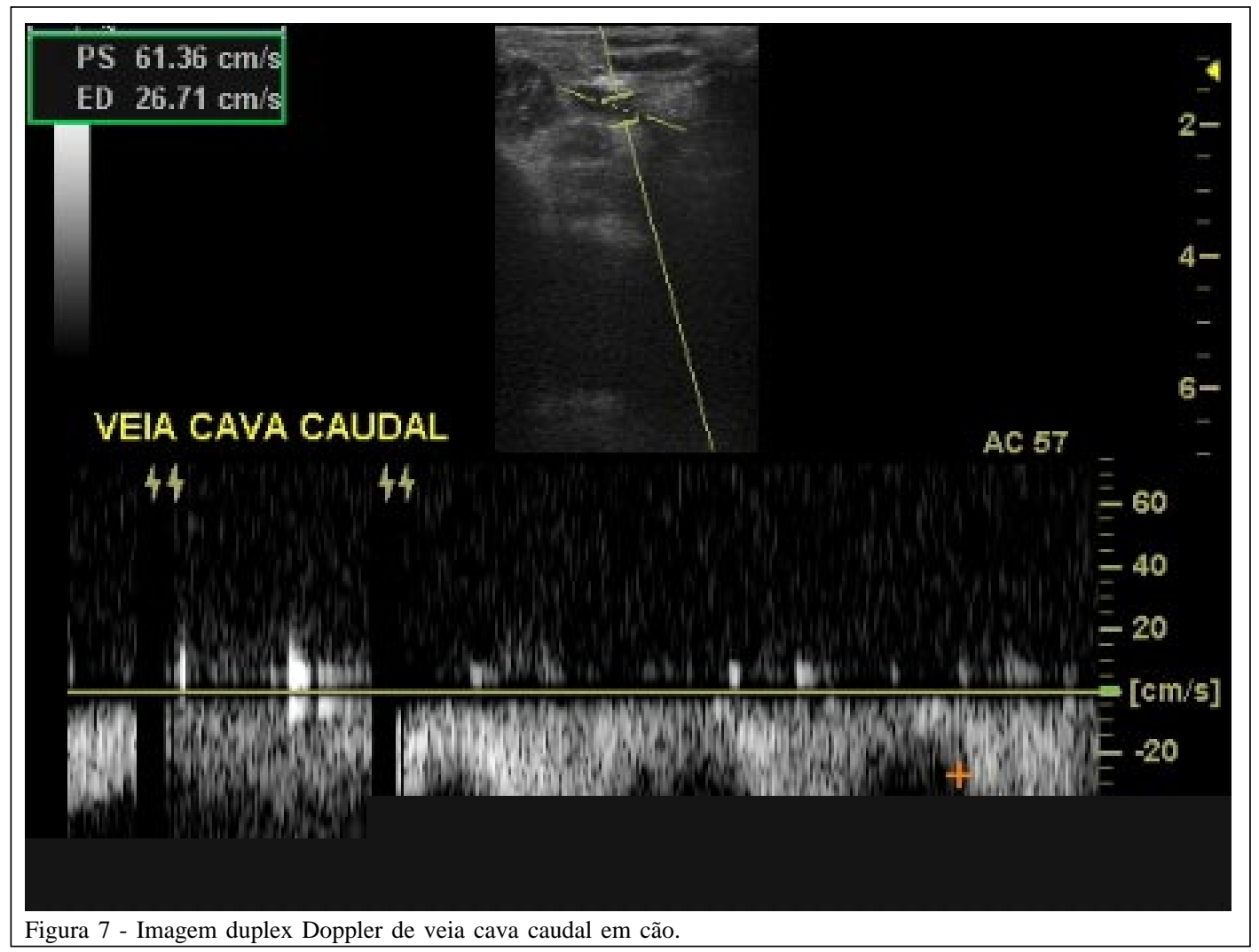

Ciência Rural, v.38, n.3, mai-jun, 2008. 


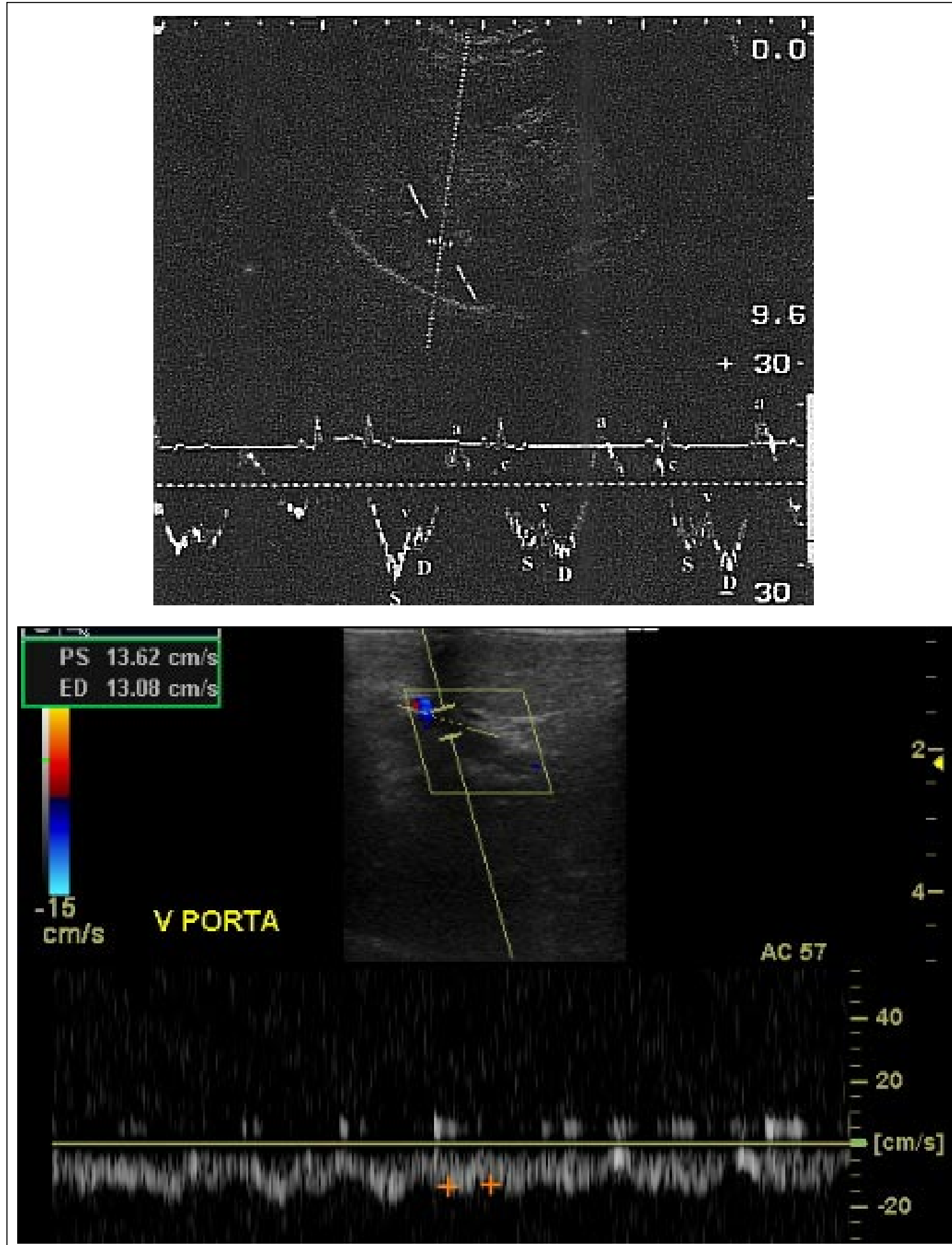

Figura 8 - A) Imagem duplex Doppler de veia hepática em cão. B) Imagem triplex Doppler de veia porta em cão.

fluxo do sangue do átrio direito para o ventrículo direito. Esta segunda onda anterógrada geralmente é menor do que a primeira. O quarto componente é a segunda onda anterógrada (onda-A), causada pela contração atrial direita. Às vezes, pode ser vista uma quinta onda, que é retrógrada (onda-C), como resultado do fechamento da valva tricúspide (CERRI et al., 1998; SZATMARI et al., 2001).

Ciência Rural, v.38, n.3, mai-jun, 2008. 
Os movimentos de inspiração podem ocasionar ligeiro aumento na onda-D e uma ligeira diminuição na onda-S, tanto no homem, quanto nos cães. As alterações de pressão intra-torácica e intraabdominal, que ocorrem devido aos movimentos respiratórios, influenciam o padrão espectral Doppler muito mais no cão do que no homem. Geralmente, durante a inspiração profunda, não é possível manter o volume de amostra no lúmen vascular. No entanto, pode-se observar que a inspiração causa aumento na velocidade de fluxo em direção anterógrada (SZATMARI et al., 2001).

\section{Veia porta}

A veia porta pode ser visualizada melhor em decúbito dorsal no plano sagital oblíquo, quando a parte anterior do transdutor está na linha branca e rotacionado ligeiramente para a direita. Esta veia nunca possui um trajeto retilíneo como o da veia cava. A veia porta principal bifurca-se na região da porta hepatis. Os ramos portais intra-hepáticos têm parede ecogênica, o que torna sua identificação mais fácil em relação às veias hepáticas (estas últimas não apresentam paredes evidentes). As paredes das veias hepáticas são hiperecóicas somente quando o feixe sonoro incidente e o vaso estão perpendiculares (KAMIKAWA et al., 2003).

Normalmente, o fluxo é hepatopetal (isto é, vem em direção ao fígado). $O$ fluxo é fásico, a velocidade é mais alta durante a expiração e mais baixa durante a inspiração (Figura 8). Provavelmente, esta alteração pode ser um artefato, que decorre da variação da localização do volume de amostra, que passa do centro do lúmen para a periferia e volta novamente de acordo com os movimentos do vaso durante a respiração (SZATMARI et al., 2001).

\section{CONCLUSÃO}

Conclui-se que o conhecimento dos traçados normais dos vasos e de suas variações auxiliam na identificação e no conhecimento da topografia dos mesmos. Assim, a identificação de estruturas anormais vasculares ou mesmo a identificação da ausência de vascularização em alguns órgãos torna-se facilitada com a ferramenta diagnóstica Doppler.

\section{REFERÊNCIAS}

CARVALHO, C.F. Ultra-sonografia em pequenos animais. São Paulo: Roca, 2004. cap.12, p.165-174.

CERRI, G.G. et al. Doppler. São Paulo: Sarvier, 1998. cap.6, p.120-121.

FINN-BODNER, S.T.; HUDSON, J.A. Abdominal vascular sonography. Veterinary Clinics of North America Small Animal Practice, v.28, n.4, p.887-941, 1998.

GASCHEN, L. et al. Pattern recognition and feature extraction of canine celiac and cranial mesenteric arterial waveforms: normal versus chronic enteropathy - a pilot study. Veterinary Journal, v.169, n.2, p.242-250, 2005.

KAMIKAWA, L. Avaliação ultra-sonográfica da aorta abdominal e seus ramos em cães. 2003. 87f. Dissertação (Mestrado em Anatomia dos Animais Domésticos) - Faculdade de Medicina Veterinária e Zootecnia da Universidade de São Paulo.

KAWAKAMA, J. et al. Física. In: CERRI, G.G.; ROCHA, D.C. Ultra-sonografia abdominal. São Paulo: Sarvier, 1993. cap.1, p.1-14.

LAMB, C.R. et al. Doppler measurement of hepatic arterial flow in dogs: technique and preliminary findings. Veterinary Radiology and Ultrasound, v.40, n.2, p.77-81, 1999.

NYLAND, T.G.; MATTON, J.S. Small animal diagnostic ultrasound. 2.ed. Philadelphia: Saunders, 2002. 461p.

NYLAND, T.G. et al. Diagnosis of urinary tract obstruction in dogs using duplex Doppler ultrasonography. Veterinary Radiology and Ultrasound, v. 34, n.5, p.384-352, 1993.

RIESEN, S. et al. Doppler measurement of splanchnic blood flow during digestion in unsedated normal dogs. Veterinary Radiology and Ultrasound, v.43, n.6, p.554-560, 2002.

SPAULDING, K.A. A review of sonographic identification of abdominal blood vessels and juxtavascular organs. Veterinary Radiology and Ultrasound, v.38, n.1, p.4-23, 1997.

SZATMÁRI, V. et al. Normal duplex Doppler waveforms of major abdominal blood vessels in dogs: a review. Veterinary Radiology and Ultrasound, v.42, n.2, p.93-107, 2001.

WU, J.X.; CARLISLE, C.H. Ultrasonographic examination of the canine liver based on recognition of hepatic and portal veins. Veterinary Radiology and Ultrasound, v.36, n.3, p.234-239, 1995.

YANIK, L. The basics of Doppler ultrasonography. Veterinary Medicine, v.3, p.388-398, 2002. 